\title{
Successful rechallenge after etoposide infusion related adverse drug reactions
}

\author{
Emma Tillman ${ }^{1}$, Sarah Suppes², Nicholas Miles ${ }^{1}$, Ashley Duty², Kelsey Kelley², and \\ Jennifer Goldman ${ }^{2}$ \\ ${ }^{1}$ Indiana University School of Medicine \\ ${ }^{2}$ Children's Mercy Hospital
}

February 12, 2021

\begin{abstract}
Etoposide is an antineoplastic agent widely used for treatment of many pediatric cancers and associated with infusion related adverse drug reactions (ADRs). In this brief report we describe etoposide infusion related ADRs that occurred over a 10-year period at two freestanding pediatric hospitals. Infusion reactions occurred in $1 \%$ of patients. Of the 32 patients that experienced adverse reactions, $41 \%$ were rechallenged after the reaction and all were able to tolerate at least one future dose with either pre-treatment or extending infusion duration.
\end{abstract}

\section{Introduction:}

Etoposide is an antineoplastic agent used in more than a dozen pediatric chemotherapeutic regimens. Case reports have described infusion related adverse drug reactions (ADRs) with etoposide. ${ }^{1-4}$ The package insert reports anaphylactic-like reactions including chills, fever, tachycardia, bronchospasm, dyspnea, and hypotension occurring in $0.7-2 \%$ of patients receiving etoposide. ${ }^{5}$ Primary literature estimates infusion reactions occur in $1.3-27.1 \%$ of patients receiving etoposide. ${ }^{6,7}$ We present a ten-year experience of etoposide, and describe infusion related reactions at two freestanding pediatric hospitals.

\section{Methods:}

After institutional review board approval, etoposide infusion related ADRs were retrospectively evaluated from January 1, 2010 - July 31, 2020 at Children's Mercy Hospital (CMH), Kansas City, MO, and Riley Hospital for Children (RH), Indianapolis, IN. Both institutions identified etoposide ADRs using multiple detection methods including ADRs entered into the electronic medical record (EMR), international classification of disease (ICD)-9 and ICD-10 codes for anaphylaxis, flushing, rash, or hypotension, orders for diphenhydramine, hydrocortisone, or epinephrine within 24 hours of etoposide dose, and orders for etoposide phosphate as this formulation is used when patients have had an etoposide ADR. These triggers prompted manual EMR review to collect data related to the ADR. Both institutions used a standard etoposide concentration of $0.4 \mathrm{mg} / \mathrm{mL}$. In-line filter use was standard protocol at CMH starting late 2017, and no filters were used at RH. Etoposide was infused over $1 \mathrm{hr}$ and $2 \mathrm{hr}$ at $\mathrm{CMH}$ and $\mathrm{RH}$ respectively, but could be modified by providers. A modified Hartwig's Severity Assessment Tool was used to classify ADR severity (TABLE 1).8,9

\section{Results:}

We identified 32 patients experiencing etoposide related ADRs (TABLE 2). Two ADRs occurred in 2012, and 30 occurred between 2017-2020. Overall, 17,134 doses of etoposide were administered to 3,326 unique patients and 32 patients (1\%) experienced ADRs. At RH, 7,489 doses of etoposide were administered to 577 
patients and three patients (0.5\%) experienced ADRs. At CMH, 9,645 doses of etoposide were administered to 2,749 patients and 29 patients (1\%) experienced ADRs.

The age of patients experiencing ADRs was 8.5 \pm 5.8 years (mean $\pm \mathrm{SD}$ ), with 17 male and 15 female patients, $27(84 \%)$ were white/non-Hispanic, 3 Hispanic (9\%), 1 black (3\%), and 1 multiracial (3\%). Fifty percent of patients had a history of a previous food or drug allergy, and $19 \%$ had a documented past medical history of an allergic or inflammatory condition.

Infusion reactions occurred with the first dose in $53 \%$ patients. Seventy-five percent were characterized as The ADR was characterized as moderate in $75 \%$ and $25 \%$ as severe reactions, yet no patients required admission to the intensive care unit and all fully recovered.

Eighty-eight percent of patients experienced multiple symptoms during the etoposide infusion, with an average of $2.8 \pm 1.1$ symptoms per patient. The most common symptoms were flushing and difficulty breathing (including chest or throat tightness) which occurred in $71 \%$ of patients. Coughing was described in $50 \%$ of patients, facial or lip swelling $45 \%$, redness or rash and nausea and vomiting were both reported to occur in $33 \%$ of patients.

Multiple treatments were administered to $59 \%$ of patients with an average of $2.2 \pm 1.5$ treatments per patient. The most common treatment was diphenhydramine which was given to $94 \%$ of patients, hydrocortisone in $25 \%$ of patients, histamine H2-receptor antagonist in $18 \%$ of patients, and IV fluids in $16 \%$ of patients. The infusion time was extended in $28 \%$ of patients,

Thirteen patients (41\%) were re-challenged with etoposide after the reaction, and all were initially able to tolerate at least one future dose with either pre-treatment, and/or extending infusion duration. However, three of the 13 patients re-challenged did subsequently experience a second reaction with etoposide resulting in a change to etoposide phosphate formulation. Nineteen patients (59\%) were never re-challenged with the standard etoposide formulation. Overall, 22 patients ultimately received etoposide phosphate and no ADRs were reported with this formulation.

\section{Discussion:}

We report a series of etoposide infusion related ADRs occurring at two free standing pediatric institutions. Specifically, our results highlight three key findings: 1) etoposide can be tolerated in patients who have experienced an etoposide ADR with premedication, extended infusion time, or change in formulation, 2) flushing and difficulty breathing were the most commonly encountered symptoms, and 3) we observed varying rates of ADRs between institutions and a clustering of etoposide infusion ADRs between 2017-2020.

Often times when a patient experiences an ADR, the patient is not re-challenged and a therapeutic alternative is prescribed. ${ }^{11}$ In cancer treatment, alternative therapeutic agents are often not an option. In our 10year cohort all 32 patients that experienced etoposide ADRs were successfully re-challenged with either etoposide or etoposide phosphate in order to complete their prescribed regimen. Sixty percent of patients were empirically changed to the etoposide phosphate formulation which has been shown to be associated with fewer ADRs compared to standard etoposide formulations, but due to the higher price, it is not typically used first-line. ${ }^{7,11}$ The remaining $40 \%$ of patients received premedication and/or modifications of infusion rates and tolerated subsequent infusions. Interestingly, no ADRs were associated with etoposide phosphate.

Etoposide infusion related ADRs are reported in both the package insert and previously published literature with a wide variety of symptomology. ${ }^{1-5,7}$ Although in our cohort we present many possible symptoms, the ADRs observed in our population are homologous with $71 \%$ of patients experiencing flushing and respiratory distress. This is important, as monitoring for these symptoms should occur during etoposide infusions.

The rate of etoposide $\mathrm{ADRs}$ at $\mathrm{CMH}$ was twice that at $\mathrm{RH}$, but more interestingly across both institutions, two ADRs occurred in 2012, one at each institution, and the remaining 30 ADRs all occurred during the fouryear period between 2017-2020, with no etoposide ADRs reported in 2010, 2011, 2013-2016. This clustering prompted both centers to evaluate potential differences in practice between our institutions and during 
different time periods. Two distinct differences between institutional standard practices are infusion time and use of an in-line filter. At RH the standard infusion time for etoposide is $2 \mathrm{hrs}$ and the standard infusion time at $\mathrm{CMH}$ is $1 \mathrm{hr}$. After extending the infusion time, $28 \%$ of $\mathrm{CMH}$ patients were able to tolerate future doses of etoposide. Rate of infusion has previously been associated with ADRs, as faster rates result in more ADRs. ${ }^{7}$ Additionally, the use of an in-line filter at $\mathrm{CMH}$ was standard of care to prevent precipitation starting in late 2017 and this was not used at RH. The presence or absence of a filter has not been discussed in previous reports of etoposide ADRs. In-line filters and faster infusion rates are potential risk factors for etoposide ADRs and evaluation of practice across institutions could be informative to determine strategies to minimize ADR risk.

Due to the retrospective nature of this study, it is possible that etoposide ADRs could have been missed, however multiple approaches were used to identify ADRs. Additionally, etoposide manufacture and lot numbers were not available; therefore, we were unable to determine any link to specific products and ADR. Our work highlights the importance of looking across sites at ADRs as variation in practice can provide key information about ADR phenotype and potential risk factors emphasizing the critical need for a systematic approach to identifying ADRs trends across institutions. Future etoposide ADRs may be prevented or quickly identified by implementing slower infusion times, standard premedication, and close monitoring for during infusions.

\section{Conflict of Interest statement:}

No authors have any conflicts of interest to report.

\section{Acknowledgements:}

None

\section{References:}

1. de Souza P, Friedlander M, Wilde C, Kirsten F, Ryan M. Hypersensitivity reactions to etoposide. A report of three cases and review of the literature. Am J Clin Oncol 1994;17:387-9.

2. Hoetelmans RM, Schornagel JH, ten Bokkel Huinink WW, Beijnen JH. Hypersensitivity reactions to etoposide. Ann Pharmacother 1996;30:367-71.

3. Kasperek C, Black CD. Two cases of suspected immunologic-based hypersensitivity reactions to etoposide therapy. Ann Pharmacother 1992;26:1227- 30.

4. Ogle KM, Kennedy BJ. Hypersensitivity reactions to etoposide. A case report and review of the literature. Am J Clin Oncol 1988;11:663-5.

5. Etoposide. Package insert. Fresenius Kabi USA, LLC; 2019.

6. Turgay Yagmur I, Guzelkucuk Z, Yarali N, et al. Evaluation of hypersensitivity reactions to cancer chemotherapeutic agents in pediatric patients. Ann Allergy Asthma Immunol 2020;124:350-6.

7. Stockton WM, Nguyen T, Zhang L, Dowling TC. Etoposide and etoposide phosphate hypersensitivity in children: Incidence, risk factors, and prevention strategies. J Oncol Pharm Pract 2020;26:397-405.

8. Hartwig SC, Siegel J, Schneider PJ. Preventability and severity assessment in reporting adverse drug reactions. Am J Hosp Pharm 1992;49:2229-32.

9. Yu D, Sheets J, Suppes S, Goldman J. Characterization of Severe Adverse Drug Reactions at a FreeStanding Children's Hospital. J Clin Pharmacol 2019;59:1569- 72.

10. Meyboom R. Intentional rechallenge and the clinical management of drug-related problems. Drug Saf $2013 ; 36: 163-5$.

11. Collier K, Schink C, Young AM, How K, Seckl M, Savage P. Successful treatment with etoposide phosphate in patients with previous etoposide hypersensitivity. J Oncol Pharm Pract 2008;14:51-5. 


\section{Hosted file}

Table 1.pdf available at https://authorea.com/users/395353/articles/508644-successfulrechallenge-after-etoposide-infusion-related-adverse-drug-reactions

Hosted file

Table 2.pdf available at https://authorea.com/users/395353/articles/508644-successfulrechallenge-after-etoposide-infusion-related-adverse-drug-reactions 